gene expression: genes closest to H3K27me3 group dACRs were typically silenced, whereas those closest to H3Kac group dACRs were usually upregulated. $\mathrm{Hi}-\mathrm{C}$ and HiChIP analyses confirmed that dACRs made direct contact with target genes via chromatin loops, with 'active' and 'silent' dACRs interacting with expressed and unexpressed genes, respectively.

Importantly, Lu et al. reported similar dACR groups from their analysis of 13 diverse angiosperm genomes, including maize. Moreover, they found that histone modifications at dACRs were conserved across species, suggesting these groups are functionally important.

The species analysed by Lu et al. included both monocots and dicots, with genomes ranging in size from $\sim 100-5,000 \mathrm{Mb}$. The total number of dACRs detected by ATAC-seq was largely consistent across species irrespective of genome size. However, the proportion of dACRs relative to proximal ACRs (pACRs; those
$<2 \mathrm{~kb}$ from a gene) were clearly correlated with increased genome size. Many dACRs were conserved across species, which allowed orthologous pairs to be identified and investigated in species with different genome sizes. These analyses indicated that transposon activity increased the distance between ACRs and their target genes in species with larger genomes, leading to a higher proportion of $\mathrm{dACRs}$ relative to pACRs in large genomes.

Taken together, these studies indicate that dACRs are widespread in angiosperm genomes and particularly prevalent in large genomes. Many dACRs are likely to be functional CREs, with distinct chromatin properties that are predictive of, and important for, their gene regulatory functions.

Dorothy Clyde

ORIGINAL ARTICLES Ricci, W. A. et al.

Widespread long-range cis-regulatory elements in the maize genome. Nat. Plants https://doi.org/ 10.1038/s41477-019-0547-0 (2019) | Lu, Z. et al. The prevalence, evolution and chromatin signatures of plant regulatory elements. Nat. Plants https://doi.org/10.1038/s41477-019-0548-z (2019)

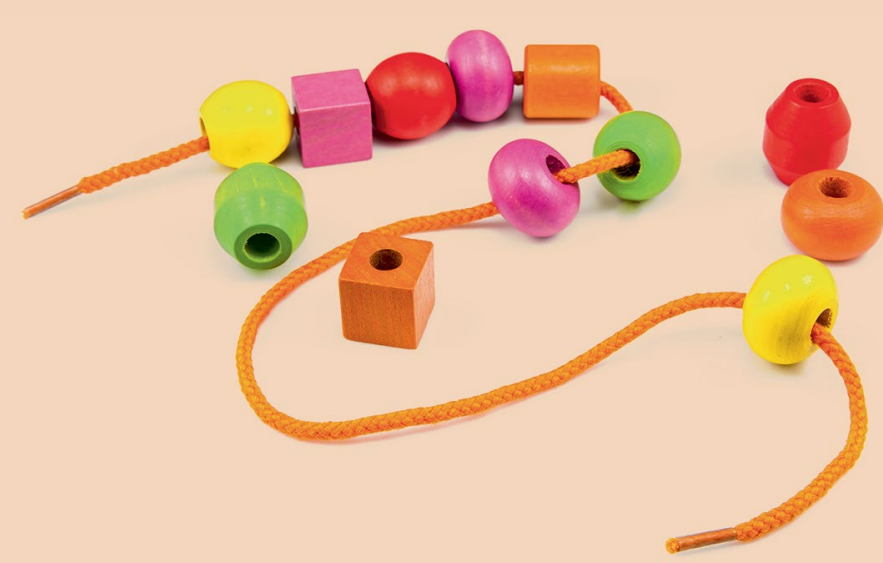

splicing factors, including HNRNPU, affected TSS use across hundreds of genes. Protein-protein interaction data from the STRING database showed the proteins contributing to the biggest changes in TSS use interacted with a network of 65 other proteins, including subunits of RNAPII and general transcription factors. Gene ontology term analysis showed EMATS was most commonly found in genes associated with synapse assembly and brain and neuron development, suggesting a role for
EMATS in regulating neurological development.

Although the exact mechanism for EMATS is unclear, the authors suggest it might be due to direct positive regulatory effects of splicing machinery on the recruitment of transcription factors to upstream promoters.

Joseph Willson

ORIGINAL ARTICLE Fiszbein, A. et al.

Exon-mediated activation of transcription starts. Cell https://doi.org/10.1016/j.cell.2019.11.002 (2019)

\section{REGULATORY ELEMENTS}

\section{Contact maps and brain disease risk}

The majority of disease-associated genetic variation resides in non-coding regions of the genome, which holds true for neurological and psychiatric disorders; many disease-causing variants exert their effects by affecting gene expression regulation. A new study in Science has mapped regulatory elements for major cell types of the human brain to help elucidate the transcriptional mechanisms underlying their developmental and functional properties in health and disease.

Using fluorescence-activated nuclei sorting, Nott et al. isolated astrocyte, microglial, neuronal and oligodendrocyte nuclei from resected cortical brain tissue of six different individuals and performed assay for transposase-accessible chromatin sequencing (ATAC-seq) to identify open chromatin regions in 200,000 cell type-specific nuclei. Moreover, 500,000 nuclei were analysed by chromatin immunoprecipitation followed by sequencing (ChIP-seq) for the histone 3 marks lysine 27 acetylation ( $\mathrm{H} 3 \mathrm{~K} 27 \mathrm{ac}$ ) and lysine 4 trimethylation (H3K4me3) to pinpoint active enhancers and promoters, respectively, in each cell type. The authors found a "oneto-many relationship between promoters and enhancers", as previously identified. In contrast to active promoters, most of which were shared across cell types, the fraction of active enhancers that overlapped between different cell types was fairly small. This finding is indicative of cell-type specificity being modulated predominantly by the enhancer repertoire.

Linkage disequilibrium score regression was applied to genome-wide association study (GWAS) summary statistics to estimate single-nucleotide polymorphism-based heritabilities, identifying an association between neuron-specific variants in transcriptional enhancers or promoters and psychiatric disorders or behavioural traits. By contrast, Alzheimer disease risk variants were found to be most prominent in microglia-specific enhancers.

Proximity ligation-assisted ChIP-seq (PLAC-seq), in which active promoters are enriched by $\mathrm{H} 3 \mathrm{~K} 4 \mathrm{me} 3 \mathrm{ChIP}$-seq after a proximity ligation step, mapped chromatin loops between active promoters and distal regulatory regions in microglia, neurons and oligodendrocytes, leading to the identification of 219,509 significant interactions across cell types. This interactome information was able to support the interpretation of disease risk variants threefold: by linking risk variants to more distal active promoters; by extending the identification of genes linked to enhancers harbouring risk variants beyond genes known from GWAS; and by identifying cell type-specific enhancers harbouring risk variants linked to genes expressed in multiple cell types, suggestive of cell type-specific disease susceptibility.

These brain cell type-specific enhancer-promoter interactome maps will serve as a useful resource to identify genes that influence neurological and psychiatric disorders and the cell types in which they function.

Linda Koch

ORIGINAL ARTICLE Nott, A. et al. Brain cell type-specific enhancer-promoter interactome maps and disease-risk association. Science 366, 1134-1139 (2019) RELATED ARTICLE Schoenfelder, S. \& Fraser, P. Long-range enhancer-promoter contacts in gene expression control. Nat. Rev. Genet. 20, 437-455 (2019) 